\title{
ABORDAJE CIENTÍFICO DE LA EDUCACIÓN SEXUAL EN ESTUDIANTES UNIVERSITARIOS
}

SCIENTIFIC APPROACH TO SEX EDUCATION IN UNIVERSITY STUDENTS

\author{
Raquel Olivia De los Santos de Dios¹, María Lyssette Mazó Quevedo², Fabiola Torres Méndez ${ }^{3}$, \\ Rocío Guadalupe Sosa Peña ${ }^{4}$ \\ Universidad Popular de la Chontalpa, Cárdenas, Mexico \\ 1. Email: raquel270876@hotmail.com (D) https://orcid.org/0000-0002-1341-657X \\ 2. Email: mlmazoquevedo@upch.mx (D) https://orcid.org/0000-0001-9174-3704 \\ 3. Email: fabiola.torres@upch.mx (D) https://orcid.org/0000-0002-0180-652X \\ 4. Email: rocio.sosa@upch.mx (iD https://orcid.org/0000-0003-4949-9984
}

Como Citar: De los Santos de Dios, R. O., Mazó Quevedo, M. L., Torres Méndez, F., \& Sosa Peña, R. G. (2021). Scientific approach to sex education in university students. Revista Publicando, 8(29), 87-93. https://doi.org/10.51528/rp.vol8.id2168

\begin{abstract}
RESUMEN:
La educación sexual, un medio importante para la formación de los jóvenes estudiantes que debe ser pertinente para sus experiencias sexuales de forma consciente, responsable y segura. El artículo presenta un estado del arte sobre la producción científica en materia de educación sexual en estudiantes universitarios en los últimos cinco años. El diseño de la investigación fue descriptivo y cuantitativo de una muestra de 90 artículos seleccionados que se enfocaron a la educación sexual en general o a dimensiones específicas de la sexualidad y el conocimiento que los estudiantes universitarios tienen de estas. Los resultados muestran que las publicaciones científicas se han orientado hacia investigaciones transversales basadas en la confirmación de hipótesis, por lo que se recomienda abrir futuras líneas de investigación proyectiva para el diseño e implementación de proyectos enfocados a la educación sexual universitaria.
\end{abstract}

\section{PALABRAS Clave:}

Estado del arte, Educación sexual en estudiantes universitarios, Formación universitaria integral.
Recibido: $08 / 12 / 2020$

Aceptado: 27/03/2021

\section{ABSRTACT:}

Sexual education is an important element in young student's formation, it must be pertinent to develop conscious, responsible, and safe sexual experiences. This article presents a state of art about scientific production in sexual education among university students in the last five years. The design of this study was descriptive and quantitative, the sample consisted of 90 scientific papers which are focused on sexual education in a general way or on specific dimensions of sexuality and the knowledge university students have about these. The results show that the scientific papers are oriented to transversal research and are based on the confirmation of hypothesis. Consequently, it is recommended to open new lines of research for the design and implementation of projects, to focus on sexual education at university level.

\section{KEYWORDS:}

State of the art, Sexual education on university student's, integral university formation. 
궁

$\begin{array}{lllllll}R & \mathrm{E} & \mathrm{V} & \mathrm{I} & \mathrm{S} & \mathrm{T} & \mathrm{A}\end{array}$

P U B L I C A N D O

I S S N $133900-93304$

\section{INTRODUCCIÓN}

$\mathrm{L}$

a educación sexual permite a los individuos asumir una actitud evolutiva y positiva ante la sexualidad, bajo la premisa de que la actitud es una predisposición cognitiva del comportamiento que facilita la toma decisiones para tales situaciones. Morales (2007), señala que una actitud sexual en los jóvenes tendría una influencia importante sobre las decisiones y procedimientos frente a las situaciones relacionadas con la sexualidad.

Tomando en cuenta lo antes expuesto, la Federación Internacional de Planificación de la Familia (IPPF) (Citado en Chávez, Petrzelová y Zapata, 2009), acota que la sexualidad es un estado general de bienestar integral que involucra los procesos del sistema reproductivo, y de la misma manera lo que la Organización Mundial de la Salud (OMS) define (Citado en Priego y Cosme, 1995), que la salud sexual se entiende como la integración de aspectos corporales, emocionales, intelectuales y culturales, para el desarrollo de la personalidad, la comunicación y una sexualidad realizada, y aunado a lo encontrado por Ordoñez, Real, Gallardo, Alvarado y Roby (2017) que los valores y comportamientos individuales como grupales, son construidos por la sociedad, y que estos son los factores que permiten la creación de una cultura evolutiva sobre la responsabilidad, el cuidado y el desarrollo social: la salud sexual es una condición de plenitud personal resultante de un proceso social constructivo y aplicativo de valores culturales orientados a los cuidados y responsabilidades correspondientes al desarrollo humano.

El lento avance histórico de la educación sexual escolar ha propiciado que en la actualidad los jóvenes asuman posturas triviales ante la sexualidad, esta falta de educación ha influido en una tasa de incremento en la adquisición de infecciones y complicaciones de salud que reducen sus posibilidades de desarrollo personal.Según la OMS (2015) de 135 millones de partos anuales, 20 millones presentan complicaciones y 830 mujeres mueren diario en el mundo por dichas complicaciones, entre ellos jóvenes (OMS, 2019); en razón de que el 50.5\% de la población lo constituyen personas menores de 30 años de edad (International Labour Office, 2012) y el $99 \%$ de la mortalidad materna ocurre en países en desarrollo (OMS, 2019).

México es de los países donde la sexualidad aún es un tema abordado con dificultades dentro de los hogares y las escuelas, en virtud de que persisten los prejuicios, esto contribuye a que los jóvenes de este país desconozcan el uso formal de métodos de prevención; por lo que es de los principales países en el ranking de embarazos y contagios del Síndrome de Inmunodeficiencia Adquirida (SIDA) en adolescentes. Según los índices de la Estrategia Nacional para la Prevención del Embarazo en Adolescentes, ENAPEA, el $45 \%$ de las mujeres de 15 a 19 años no utilizó anticonceptivos, además el $42.1 \%$ de los embarazos se ha presentado en mujeres menores de 17 años (citado en Secretaría de Salud, 2018), el 19.25\% de los nacimientos registrados en 2017 se dio en menores de 19 años (CONAMED, 2019), y siete de cada 10 mujeres en edad fértil han usado algún método anticonceptivo (Instituto Nacional de Estadística y Geografía, 2018).

Por su parte, el Programa Conjunto de las Naciones Unidas sobre el VIH/SIDA (ONUSIDA) (citado en González, 2017), reportó a México como el quinto país de Latinoamérica en casos de SIDA. Según Ortiz y Macelly, los estados más afectados en estos índices son: Campeche, Guerrero, Quintana Roo, Tabasco y Veracruz (citado en University of Veracruz, 2013), además, las infecciones de transmisión sexual ocurren con frecuencia entre los 15 y 49 años y hasta 2008 se registraron 10,000 casos (citado en Jiménez, 2010).

La ausencia de contenidos estructurados sobres temas de educación sexual, dentro de los planes curriculares de las instituciones educativas, y aun los que se han implementado, no han tenido el impacto esperado en los jóvenes, lo que afecta la formación integral del estudiante; quien, al desconocer las características y el funcionamiento de los procesos anatómicos y sexuales, toman decisiones que terminan por reflejarse en los índices de deserción, rezago y titulación. Chávez, Petrzelová y Zapata (2009), señalan que existe una relación entre los índices de deserción, rezago y titulación escolar con los índices de embarazos no deseados, enfermedades de transmisión sexual, y la sexualidad precoz, pues los programas de educación sexual que se han aplicado no han tenido la efectividad prevista en los últimos 40 años. La educación sexual ha de tender a un abordaje multidisciplinario para que sea efectiva; que se deriva de la necesidad de emprender acciones educativas con los adolescentes a edades cada vez más tempranas, involucrando de manera activa y decidida a los padres, maestros y personal relacionado con la salud pública. (Chávez, Petrzelová y Zapata, 2009)

Ante el poco efecto histórico que han tenido los programas implementados y la problemática social y de salud persistente, se justifica la importancia de abordar las metodologías de investigación que se han realizado sobre la educación sexual en los jóvenes universitarios como base de los programas de desarrollo. En este sentido, esta investigación tuvo como objetivo realizar un estado del arte sobre el tema de la sexualidad en universitarios, abordado en documentos localizados en la base de datos de ProQuest. 


\section{MÉTODO}

Para obtener la muestra referencial de publicaciones científicas orientadas a la educación sexual en estudiantes universitarios se partió de la base de datos bibliográfica ProQuest ya que es un recurso de colecciones electrónicas que contiene millones de artículos publicados originalmente en revistas, periódicos y publicaciones seriadas en general. Las palabras claves que se utilizaron para la búsqueda de artículos fue "educación sexual en estudiantes universitarios". Se obtuvo un universo total de 348 artículos resultantes que contenían las palabras claves con la restricción de cuatro filtros de búsqueda que se muestran en la tabla. Sin embargo, el 74\% de los artículos solo mencionaban las palabras claves y no como problemática central, por lo que a partir de una revisión pretextual se extrajo solo el $26 \%$ del total de publicaciones que si cumplieron como artículos sobre educación sexual.

\section{Tabla 1}

Filtros de búsqueda de publicaciones sobre educación sexual en universitarios

\begin{tabular}{ll}
\hline Filtros & Restricción \\
\hline Palabras claves & $\begin{array}{l}\text { "Educación sexual en estudiantes } \\
\text { universitarios" }\end{array}$ \\
Fecha de publicación & "Últimos 5 años" \\
Tipo de documento & "Articulo principal" \\
Tipo de fuente & "Revistas científicas" \\
Limitado a & "Artículos evaluados por expertos \\
& y texto completo" \\
\hline
\end{tabular}

El procedimiento para el tratamiento de la muestra consistió primero en realizar una lectura inter e intratextual para delimitar las publicaciones en el tiempo y el espacio, es decir los años y los contextos en que fueron publicados. Para cuantificar los contenidos, el análisis de contenidos estuvo basado en los tres momentos comunes de la hermenéutica: 1) contextualización, 2) clasificación y 3) categorización (Vélez y Calvo, 1992). En el proceso de contextualización se identificaron las temáticas más comunes, los planteamientos de problemas y sus delimitaciones, esto porque dentro de la muestra hubo heterogeneidad y existían investigaciones profundizadas en dimensiones distintas dentro de la educación sexual. Posteriormente se realizó el proceso de clasificación donde se determinaron los parámetros para la sistematización del análisis: objetivos de investigación, niveles de análisis y cortes temporales. En el proceso de categorización para la elaboración de los hallazgos sobre la muestra se basó en dos categorías: internas y externas. En las primeras se discutió el enfoque de las temáticas, las naturalezas de las metodologías y la identificación de estudios retrospectivos o prospectivos, mientras que en la segunda categoría se trató de identificar las contribuciones socioculturales de las investigaciones.

\section{RESULTADOS}

En la Figura 1 podemos observar el comportamiento de las publicaciones durante los últimos cinco años sobre educación sexual en estudiantes universitarios y se puede observar que entre el 2016 y 2018 hubo un incremento acelerado sobre el tema y alcanzó su punto máximo en el año 2018 como el más productivo con 26 publicaciones científicas. El año 2015 se registra como el de menor actividad sobre el tema.

\section{Figura 1}

Flujo de publicaciones de educación sexual universitaria $2015-2020$

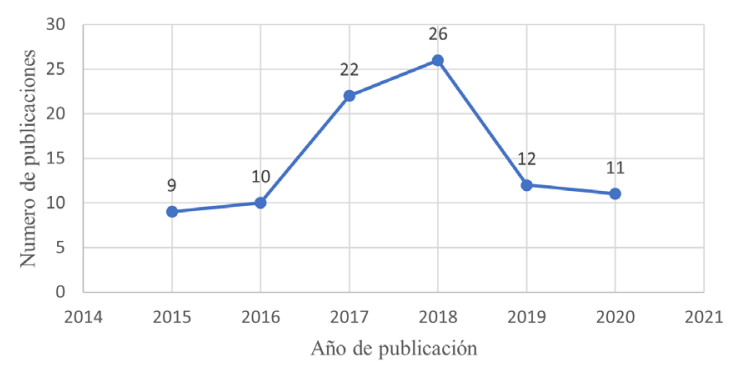

Nota: Número de publicaciones anuales sobre educación sexual en los universitarios. Elaborado por autora.

También se reportan las tendencias sobre los contextos en los que se encontró mayor interés en estos temas. Cabe aclarar que, en este apartado los resultados solo son representativos de la muestra en particular y no concluyentes, pues existen otros repositorios y metabuscadores en las que se pueden observar otros comportamientos en los resultados de búsqueda y las preferencias de publicación, en este caso se han obtenido resultados representativos del contexto latinoamericano, hispanoamericano y uno anglosajón. Colombia es el país que protagoniza la productividad en temas de educación sexual universitaria seguidos por España y Brasil. Es importante señalar que México figura entre los países con menor número de publicaciones en esta temática. 
Figura 2

Productividad en investigación de educación sexual en universitarios por países

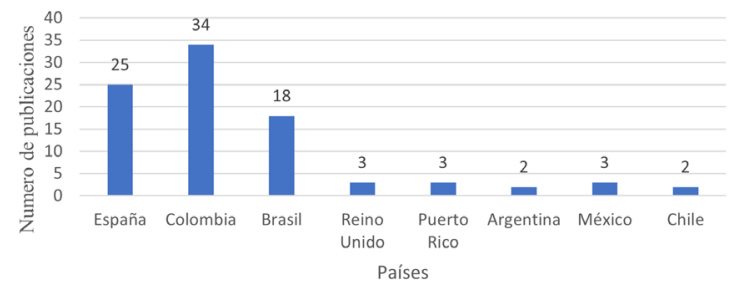

Nota: Número de publicaciones científicas de educación sexual en universitarios por países. Elaborado por autora.

En temas de educación sexual universitaria se pueden encontrar distintas temáticas específicas y relacionadas entre sí que pretenden explicar el problema de la educación sexual en forma analítica. Del total de la muestra analizada, la Figura 3 muestra que el tema más predominante es la educación sexual en general, seguido por temas específicos como Enfermedades de Transmisión Sexual (ETS) y Embarazos, se observa una diferencia mínima entre el abordaje de ETS y embarazos. Existe un interés moderado por los temas relacionados con el conocimiento de anticonceptivos y precocidad, los temas con menor interés resultaron ser abortos y promiscuidad.

\section{Figura 3}

Temáticas principales de la educación sexual en universitarios

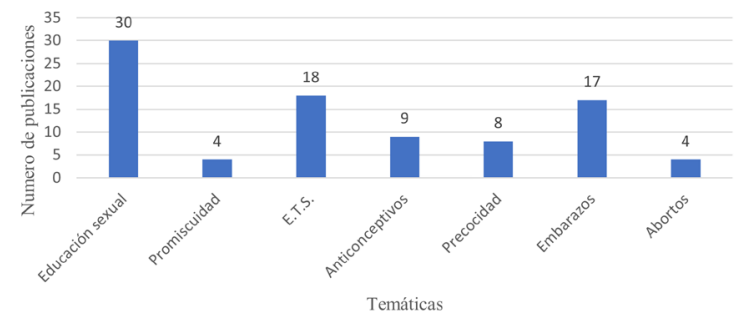

Nota: Número de publicaciones por temáticas relacionadas a la educación sexual en universitarios. Elaborado por autora.

En la Figura 4 se reporta que el 23\% de las publicaciones partió de un diagnostico relacionado con la educación sexual, seguido por el $21 \%$ que planteo el problema a partir de datos que tienen que ver con el conocimiento de los estudiantes sobre Enfermedades de Transmisión Sexual, y en tercer lugar se encuentran los artículos relacionados con los embarazos. Menor a estos, se observan los planteamientos relacionados con precocidad, abortos, deserción escolar y problemas laborales mantuvieron un interés uniforme en las publicaciones, finalmente los temas de sexismo y acoso sexual fueron los planteamientos menos predominantes.

Figura 4

Planteamientos del problema

$\begin{array}{ll} & \\ \text { - Embarazos } & \text { " Enfermedades de transmision sexual } \\ \text { - Precocidad } & \text { " Abortos } \\ \text { - Deserción } & \text { " Acoblemas laborales sexual } \\ \text { - Sexismo } & \end{array}$

Nota: Porcentaje de planteamientos de problemas típicos en las publicaciones de educación sexual. Elaborado por autora.

Con relación a los objetivos de investigación, la Figura 5 muestra que el $44 \%$ de las publicaciones se enfocaron en identificar conductas ante la sexualidad, es decir, estudios relacionados con describir variables actitudinales, que se define como elementos de un proceso de enseñanza que tienen que ver con valores, normas, creencias y actitudes, el $22 \%$ de las publicaciones se orientó a medir el conocimiento sobre la sexualidad, esto es investigaciones que buscaron confirmar un modelo cognitivo en el sujeto de estudio, el $13 \%$ pretendió identificar prácticas sexuales comunes, esto se refiere a investigaciones que buscaron comportamientos específicos en la experiencia sexual como masturbación, felación, parafilias y sus implicaciones. El 19\% de las investigaciones planteó el objetivo de identificar percepciones sobre sexualidad, es decir, basadas en la búsqueda de creencias personales del sujeto ante el fenómeno, y finalmente solo el $3 \%$ de las investigaciones se orientó en realizar un análisis histórico temporal, evolución o paradigmas de la educación sexual.

Figura 5

Objetivos de investigación

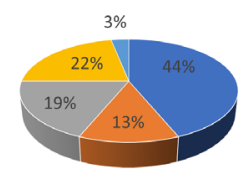

\footnotetext{
- Identificar conductas ante la sexualidad - Identificar practicas sexuales comunes - Identifcar percepciones sobre sexualidad = Medir el conocimiento sobre sexualidad - Analisis historico temporal
}

Nota: Porcentaje de objetivos de investigación típicos en las publicaciones de educacióin sexual. Elaborado por autora. 
A continuacion se presenta el comportamiento de la muestra de publicaciones con relación a los niveles de analisis. En la Figura 6 se observa que el 40\% de la mayoría de las investigaciones fueron de nivel descriptivo de las variables, el $36 \%$ fue a nivel exploratorio y el $24 \%$ fueron investigaciones de carácter correlacional principalmente cuantitativas. No se reportó dentro de la muestra investigaciones a nivel explicativo.

\section{Figura 6}

Niveles de análisis de investigación

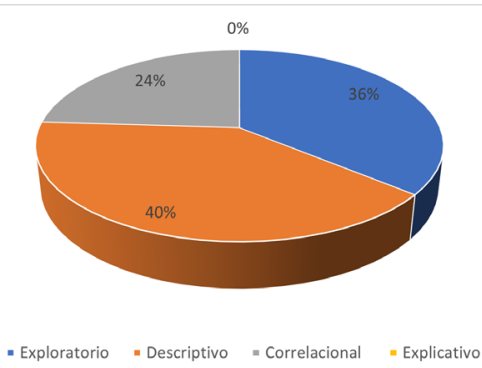

Nota: Porcentaje de los niveles de investigación típicos de las publicaciones en educación sexual. Elaborado por autora.

En la Figura 7 se muestra el corte de las investigaciones, el $92 \%$ de las publicaciones son de corte de transversal mientras que un reducido $8 \%$ fueron investigaciones de corte longitudinal.

\section{Figura 7}

Cortes temporales de las investigaciones

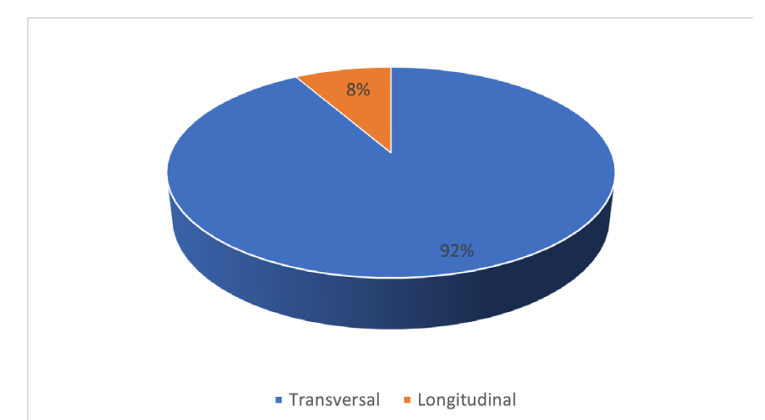

Nota: Porcentaje de cortes temporales típicos de las publicaciones de educación sexual. Elaborado por autora.

\section{DISCUSIÓN}

Históricamente el problema de la sexualidad juvenil a nivel mundial y nacional es un problema que todavía en algunos países como México no ha tenido avances significativos y las tasas de afectaciones a la salud mantienen estadísticas constantes. En contraste, la producción de investigaciones científicas sobre estos temas en los últimos cinco años mantuvo una fluctuación irregular, pues en tan solo tres años de 2015 a 2018 las investigaciones sobre la temática se triplicaron y dos años después descendieron drásticamente. También se destaca que en los distintos contextos existe cada vez más una preocupación mayor por la educación sexual, sin embargo, en el caso de México, que a pesar de ser uno de los principales países más afectados por las disfunciones sexuales no ha protagonizado productividades científicas publicables y aplicables al problema. Ordoñez et al.(2017), señalaron que la educación sexual es una responsabilidad social permanente y que está presente desde el nacimiento a través de distintos vínculos que se establecen en los círculos sociales del sujeto.

Con relación a los temas más destacados sobre la educación sexual, la aproximación al conocimiento que adoptaron las investigaciones en promedio fue a posteriori. Esto se refiere a que se orientaron más al conocimiento y actitud que tienen los jóvenes sobre los efectos posteriores a la experiencia sexual como ETS o embarazos no deseados. Si bien Chávez et al (2009) señala que una actitud sexual en los jóvenes tendría una influencia importante sobre las decisiones y procedimientos frente a las situaciones relacionadas con la sexualidad, Gorguet (2008) también argumenta que una adecuada educación sexual debería estar basada en la información sobre el manejo de anticonceptivos, la prevención de enfermedades y un conocimiento profundo sobre el tema. No se ha privilegiado el estudio a evaluar el conocimiento que poseen los jóvenes sobre la promiscuidad, la precocidad, abortos, el uso de anticonceptivos y el acoso sexual que son aspectos que están mejor relacionados con las variables explicativas del problema.

Con relación a los objetivos de investigación de las publicaciones analizadas se identificó una tendencia positivista que predominó en investigaciones basadas por hipótesis y el diagnostico. Casi la totalidad de las investigaciones buscó confirmar estructuras subyacentes como creencias, actitudes, conocimientos a través de un modelo empírico con potencia descriptiva. En poco menor medida predominaron los estudios cualitativos enfocados a la búsqueda de variables emergentes. Además, esta muestra que el índice de estudios históricos o retrospectivos fue insignificante, y la propuesta de investigaciones proyectivas o proyectos especiales para la educación sexual del alumno fue nula. Priego y Cosme (1995), argumentaron que la construcción de un modelo de educación sexual integral aportaría un equilibrio personal, habilidades comunicativas y un desarrollo de afectividad adecuado en los jóvenes. Cardona, Ariza, Gaona y Medina (2015), afirman que aún hoy, los jóvenes reciben una información incompleta o sesgada de mitos por parte 
de instituciones o personas.

\section{CONCLUSIONES}

La revisión de literatura sobre la investigación de la educación sexual en estudiantes universitarios en esta muestra de publicaciones permitió identificar áreas desarrolladas y poco desarrolladas dentro de la metodología investigativa sobre la educación sexual estudiantil, así también permitió formular sugerencias para el lector sobre la orientación que deben tomar las investigaciones y las áreas que se deben atender en este fenómeno.

Se recomienda una postura epistemológica a priori en el diseño de los modelos de investigación. Se ha demostrado con esta investigación dentro de sus delimitaciones que existen variables condicionadas confirmadas desde varios enfoques, pero también se debe proliferar el estudio de variables condicionantes o causales que están relacionadas con los orígenes del problema. Se sugiere de igual forma plantear investigaciones explicativas que aporten resultados sobre la dinámica de las variables independientes y dependientes involucradas en el fenómeno. En este mismo sentido y ante la carente investigación de estudios longitudinales e investigaciones sobre los abortos, también debe formularse hipótesis sobre investigaciones de impacto que cuestionen la relación entre la evolución de los paradigmas de la educación sexual y el fenómeno del aborto, ya que de acuerdo a los índices de abortos y muertes en sus malas prácticas no han mejorado con el tiempo.

Finalmente se reconoce que actualmente existe suficiente información científica de este tema, por tanto, se recomienda abrir nuevas líneas de investigación orientadas al desarrollo o mejoramiento de programas de intervención escolar y meta-análisis que permitan comparar las diferentes alternativas que existen para configurar una estrategia eficaz y aminorar los efectos sociales de los problemas relacionados con la sexualidad y su desconocimiento. Es importante enfatizar que se necesitan presupuestar más esfuerzos e incentivos por parte de los gobiernos, organismos e instituciones académicas de interés para mantener una tasa productiva constante en la aportación de conclusiones hacia programas de desarrollos que permitan atender con más efectividad el problema, esto se refiere a que deben estimularse las investigaciones proyectivas y diseño de proyectos especiales probados para ser implementados en los ambientes educativos. 


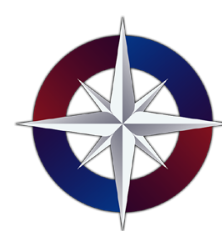

$\begin{array}{lllllll}\mathrm{R} & \mathrm{E} & \mathrm{V} & \mathrm{I} & \mathrm{S} & \mathrm{T} & \mathrm{A}\end{array}$

\section{REFERENCIAS}

Cardona, D., D.V., Ariza, G. A., Gaona, R. C., \& Medina, P. O. A. (2015). Conocimientos sobre sexualidad en adolescentes escolares en la ciudad de Armenia, Colombia. Rev.Arch Med Camagüey, 19(6), 568576.

Chávez, M., Petrzelová, J., \& Zapata, J. (2009). Actitudes respecto a la sexualidad en estudiantes universitarios. Enseñanza e Investigación en Psicología, 14(1), 137-151.

Comisión Nacional de Arbitraje Médico (2019). Panorama de la mortalidad materna adolescente en México. (Boletín CONAMED Publicación No. 25). Recuperado de: https://bit.ly/31S8dwo

Fernández, B. F., Colunga, R. C., González, S. M., Aranda, G. M. I., Riego, A. N. A., Sánchez, E. A., Enríquez, H. C. B., \& Balderrama, T. J. A. (2013). Nivel de conocimientos sobre sexualidad enestudiantes de carreras de Ciencias de la Salud de laUniversidadVeracruzana. UniverSalud, 9(17), 12-18.

González, C. H. (1 de diciembre de 2017). México, quinto lugar en AL con más casos de VIH/Sida: ONUSIDA. Recuperado: https://www.excelsior.com.mx/ nacional/2017/12/01/1204894

Gorguet, P. I. (2008). Comportamiento sexual humano. Santiago de Cuba: Editorial Oriente.

Instituto Nacional de Estadística y Geografía. (2018). Encuesta Nacional de la Dinámica Demográfica. principales Resultados. Recuperado: https://www.inegi. org.mx/contenidos/programas/enadid/2018/doc/ resultados_enadid18.pdf

International Labour Office (2012). Tackling the youth employment crisis: A macroeconomic perspective. Recuperado: https://bit.ly/2PVJgO4

Jiménez, M. (2010). Comunicación sexual en adolescentes y su implicación en la consistencia del uso del condón. Enseñanza e Investigación en Psicología, 15(1), 107-109.

Lameiras, M., \& Carrera, M. V. (2009). Educación Sexual. De la teoría a la práctica. Madrid: Pirámide

Ordoñez, S. J., Real, C. J., Gallardo, L. J., Alvarado, F. H., \& Roby, A. A. (2017). Conocimientos sobre salud se- xual y su relación con el comportamiento sexual en estudiantes universitarios. An Fac med, 78(4), 419423. https://doi.org/10.15381/anales.v78i4.14264

Organización Mundial de la Salud (2015). Datos sobre salud materna. Recuperado: https:/www.who.int/ features/factfiles/maternal_health/maternal_health_facts/es/index $2 . h t m l$

Organización Mundial de la Salud (2019). Mortalidad Materna. Recuperado de: https://www.who.int/es/ news-room/fact-sheets/detail/maternal-mortality

Priego, T., \& Cosme, P. (1995). Comprender la sexualidad para una orientación integral. Madrid: Aprender.

Secretaría de Salud. (18 de abril de 2018). Embarazo en niñas y adolescentes. Un fenómeno multifactorial. [Mensaje en un Blog]. Recuperado de: https://www. gob.mx/salud/articulos/embarazo-en-ninas-y-adolescentes

University of Veracruz. (2013). Nivel de conocimientos sobre sexualidad en estudiantes de carreras de Ciencias de la Salud de la Universidad Veracruzana. UniverSalud, 9(17), 12-18.

Vélez, A., \& Calvo, G. (1992). La investigación documental. Estado del Arte y del conocimiento. Análisis de la investigación en la formación de investigadores. Maestría en Educación. Bogotá: Universidad de la Sabana. 